\section{Informationsmanagement und Stakeholderdialog im Bauwesen}

\author{
von Martina Klingele, ITC-ZTS, und Thomas \\ Lützkendorf, Universität Karlsruhe
}

Gebäude und bauliche Anlagen sind eine wichtige und unverzichtbare Lebensgrundlage. In den Ländern Europas werden ca. 30 Prozent der Ressourceninanspruchnahme und der Wirkungen auf die Umwelt (Treibhauseffekt, Ozonschichtabbau, Versauerung von Boden und Wasser, Eutrophierung, Sommersmog) durch die Herstellung, Errichtung und Bewirtschaftung von Gebäuden verursacht. Durch eine gezielte Beeinflussung der Planung, Errichtung und Bewirtschaftung können als Beitrag zu einer nachhaltigen Entwicklung erhebliche Potenziale zur Reduzierung der Energieund Stoffströme erschlossen werden. Basis und Hilfsmittel dafür ist das Life Cycle Assessment (LCA). Bedingt durch die Besonderheiten der Bau- und Immobilienwirtschaft wird eine Integration des LCA in Entscheidungsprozesse unterschiedlichster Akteure erforderlich. Im Beitrag wird diskutiert, ob und inwieweit es bereits gelungen ist, diesen Prozess durch Bereitstellung geeigneter Daten, Hilfsmittel und Vorgehensweisen zu unterstützen, wie sich die Nachfrage nach LCA-Ergebnissen im Baubereich entwickelt und welche noch zu lösenden Fragestellungen identifiziert werden können.

\section{Einleitung}

Die Sicherung eines positiven Beitrages der Bau- und Immobilienwirtschaft $\mathrm{zu}$ einer nachhaltigen Entwicklung erfordert die Anpassung ihrer Prinzipien und Managementregeln (vgl. Rogall 2004) an den jeweiligen Betrachtungsgegenstand (u. a. Bauprozess, Bauprodukt, Bauwerk, Siedlung, Unternehmen, Bedürfnisfeld Bauen und Wohnen) sowie deren Übersetzung in den konkreten Arbeits- und Verantwortungsbereich der beteiligten Akteure. Hierfür ist es u. a. notwendig und sinnvoll,

Grundlagen zur Beurteilung des Beitrages $\mathrm{zu}$ einer nachhaltigen Entwicklung in Methoden, Instrumente und Entscheidungsabläufe der
Beteiligten $\mathrm{zu}$ integrieren, die nicht nur im engen, sondern auch im erweiterten Sinne an der Planung, Errichtung, Nutzung und Bewirtschaftung von Gebäuden beteiligt sind. Die sehr heterogen und arbeitsteilig organisierte Bau- und Immobilienwirtschaft mit ihren vielen Partnern, die sich entlang der Wertschöpfungsketten formieren, erfordert dabei u. a. die Sicherung eines Informationsflusses zwischen den jeweiligen Parteien. Dies ist auch und insbesondere für die Beschreibung und Bewertung von Energie- und Stoffströmen unter Verwendung von Methoden des Life Cycle Assessments notwendig. Neben der inhaltlichen Weiterentwicklung von methodischen und bewertungstheoretischen Grundlagen des LCA gewinnen damit zunehmend Fragen des Informationsmanagements an Bedeutung.

Methoden des Life Cycle Assessments wurden in der Vergangenheit auf einzelne, sehr grundsätzliche Fragestellungen angewendet, die sowohl wissenschaftlicher Natur waren als auch der Politik für Richtungsentscheidungen dienten. Die meist nur auf der Untersuchung von Einzelparametern (z. B. dem kumulierten Energieaufwand) basierenden Studien dienten $u$. a. dem Nachweis der ökologischen Vorteilhaftigkeit der Verbesserung der energetischen Qualität von Bauwerken in Richtung Niedrigenergieund Passivhaus, einer zusätzlichen Wärmedämmung im Bestand oder der Nutzung von Solarenergie. Planungs- und Bewertungshilfsmittel mit integriertem LCA wurden zwar entwickelt und auf dem Markt angeboten, stießen aber auf wenig Interesse. In der Vergangenheit setzten die Art der Ausbildung von Akteuren, die mangelnde Sensibilisierung gegenüber Umweltthemen sowie das Fehlen von Konsequenzen bzw. positiven Auswirkungen eines nachweislich umwelt- und gesundheitsgerechteren Planens, Bauens und Betreibens der Verbreitung des LCA in der vollen Breite des Marktes Grenzen.

Diese Situation beginnt sich zu verändern. Alle Akteure sind durch Themen wie Ressourcenverknappung und Klimawandel zunehmend sensibilisiert und bereit, ihren Beitrag zu einer nachhaltigen Entwicklung durch verstärkte Wahrnehmung ihrer Verantwortung gegenüber Umwelt und Gesellschaft zu leisten. In den Universitäten und Forschungseinrichtungen beschäftigen sich neue Gruppen von Anwendern mit Methoden des LCA. Neben der Weiterent- 
wicklung von Daten- und Bewertungsgrundlagen, bei denen methodische Fragen im Vordergrund standen und weiterhin stehen, möchten Wissenschaftler das LCA zunehmend als weiteren Aspekt und als zusätzliches Kriterium in die Produkt-, Konstruktions- und Gebäudeoptimierung integrieren. Planer sehen das LCA als ein Hilfsmittel, welches ihnen anwendungsbereit, interpretierbar und richtungssicher zur Verfügung stehen muss. Auch sonstige Akteure (wie Investoren, Rating-Agenturen, Banken und Versicherungen) beginnen sich für Ergebnisse des LCA zu interessieren. Ausgehend vom Thema Energieeffizienz soll die Frage beantwortet werden, mit welcher Ressourceninanspruchnahme und Umweltbelastung die Errichtung und der Betrieb der Gebäude verbunden sind. Dazu gehört auch die Frage, ob und welche Risiken für Umwelt und Gesundheit auftreten. Insofern wächst auch die Nachfrage nach gesicherten Bewertungsergebnissen. In Deutschland wird die Entwicklung aktuell u. a. durch das Bundesministerium für Verkehr, Bau und Stadtentwicklung (BMVBS) vorangetrieben. Nachdem mit dem Leitfaden für Nachhaltiges Bauen Orientierungen für die Planung von Bundesbauten gegeben wurden (BBR 2001), wird nun an der Entwicklung eines Zertifizierungssystems zur Beschreibung, Bewertung und Darstellung der Nachhaltigkeit von Hochbauten gearbeitet. Im Bereich Umweltqualität wird das System - soweit möglich - auf quantitativen Aussagen basieren, die sich auf Ergebnisse des LCA abstützen. Hier erfolgt ein Übergang von der Methodenentwicklung und von Fallstudien zur Untersuchung von Einzelgebäuden mit wissenschaftlich abgesicherten Methoden/Modellen als Vorbereitung von Richtungsentscheidungen hin zur Integration von Nachhaltigkeitsaspekten in konkrete Planungs-, Bewertungs- und Entscheidungsprozesse. Ergebnisse des LCA können damit u. a. Förderentscheidungen, die Wertermittlung oder den Nachweis der Erfüllung von politischen Zielen beeinflussen. Dieser Prozess führt u. a. zu veränderten Anforderungen an Planer und an die Planung - hier auch im Sinne der Bereitstellung von Bewertungsergebnissen sowohl in der Planung (Konstruktionsoptimierung) als auch im Sinne des Signalisierens von Qualität gegenüber Dritten (Zertifizierung).

\section{Stakeholder und ihre Informations- bedürfnisse}

Die Akteure im Bauwesen unterscheiden sich einerseits hinsichtlich ihrer Stellung und ihrer direkten / indirekten Rolle in den Wertschöpfungsketten (u. a. Produzent, Konsument, Berater in den Prozessen der Herstellung von Bauprodukten und der Bereitstellung von Dienstleistungen, der Planung, Errichtung und Bewirtschaftung von Gebäuden bzw. in der Initiierung, Finanzierung, Vermarktung von Objekten oder der Schaffung von Rahmenbedingungen). Andererseits sind auch ihre Motive sowie individuellen und institutionellen Ziele, ihr Informationsbedarf sowie ihre Funktion in der Informationsverarbeitung (Bereitstellung, Aufbereitung, Weitergabe bzw. Nachfrage) unterschiedlich und müssen jeweils spezifisch berücksichtigt werden.

Es handelt sich dabei zunächst um die Akteure aus den Bereichen Bauindustrie und Baugewerbe, die das LCA zum Optimieren von Produkten und Prozessen und zur Beschreibung der Umwelteigenschaften einsetzen (können) und die ihrerseits umweltrelevante Informationen aufbereiten (lassen) sowie Dritten zur Verfügung stellen (z. B. in Form von environmental product declarations). Weiterhin sind es die Investoren, Rating-Agenturen, Banken und Versicherungen, die Umweltkriterien für die eigenen Kaufentscheidungen oder das Immobilienrating heranziehen wollen und somit als indirekte Datennachfrager anzusehen sind. Schließlich sind noch der Gesetzgeber, die Normungsgremien oder die öffentliche Hand zu nennen, deren Interesse in der Entwickelung neuer gesetzlicher und normativer Anforderungen liegt, für welche Life-Cycle-Daten unterschiedlicher Aggregationsstufen Grundvoraussetzung sind.

Eine wesentliche Aufgabe an der Schnittstelle von Datennachfrage und Informationsbereitstellung erfüllen die Planenden der Bauwerke. Sie sind in der Wertschöpfungskette des Gebäudes die Ersten, die den gesamten Lebenszyklus überschauen können und als Treuhänder ihrer Auftraggeber entweder Planungsentscheidungen selbst treffen oder zumindest die Entscheidungsgrundlagen für die Bauherren aufbereiten müssen. Dies betrifft auch umweltrelevante Fragestellungen, die sich u. a. auf LCA-Ergebnisse abstützen können. 


\subsection{Planer und LCA - Erkenntnisse aus einer Online-Umfrage}

Möglichkeiten und Grenzen der Anwendung des LCA im Planungsprozess wurden in der Fachwelt kontrovers diskutiert. ${ }^{1}$ Vor diesem Hintergrund führte die Zentralabteilung Technikbedingte Stoffströme des Instituts für Technische Chemie (ITC-ZTS) am Forschungszentrum Karlsruhe 2004 im Rahmen der Aktivitäten der Abteilung im deutschen Netzwerk Lebenszyklusdaten unter dem Titel „Umweltaspekte und Ökobilanzen in der Gebäudeplanung - Umfrage unter Architekten" eine deutschlandweite Online-Umfrage durch. Die Umfrage wurde abgestimmt mit der Bundesarchitektenkammer, in deren zuständigem Fachgremium diskutiert und im Portal des Netzwerks Lebenszyklusdaten zugänglich gemacht (Klingele, Jeske 2007). ${ }^{2}$

Schwerpunkte der Fragestellung waren

- die Berücksichtigung von Umweltschutzaspekten in der Planung von Gebäuden und die Rolle, die Lebenszyklusdaten dabei zukommt,

- die Wichtigkeit der unterschiedlichen Lebensphasen eines Gebäudes im Hinblick auf seine Umweltauswirkungen,

- der Bedarf an Deklarationen von bestimmten Bauproduktgruppen und die von den Planern gewünschte Informationstiefe,

- der Bekanntheitsgrad und die Akzeptanz von LCA als Methode zur Entscheidungsfindung im Planungsprozess,

- die Planungstools, welche die Entscheidungsfindung unter Berücksichtigung der Umweltwirkungen und der Kosten von Bauwerken in allen Lebensphasen unterstützen.

Als wesentliche Erkenntnisse, die aus den Antworten zu der Umfrage abgeleitet werden können, sind zu nennen:

a) Die Berücksichtigung ökologischer Kriterien sind nach Meinung der befragten Planer wichtig; die Hälfte erklärt sogar aus Überzeugung, ohne Vergütung einen Mehraufwand in der Planung aufgrund ökologischer Anforderungen auf sich $\mathrm{zu}$ nehmen. Jedoch dürfte diese Bereitschaft zu freiwilligen Leistungen schnell an ihre Grenzen stoßen, wenn der Aufwand als zu hoch empfunden würde.

b) Aus den Umfrageergebnissen geht andererseits hervor, dass der oben erwähnten Be- reitschaft eine deutliche Unkenntnis über die Methode des LCA und der verfügbaren Software im Bereich des Bauwesens gegenüber steht. Etwa drei Viertel der Antwortenden kennen keine Ökobilanzen mit Bezug zur Architektur und mehr als die Hälfte keine LCA-Tools.

c) Nur ein Siebtel der Antwortenden der Umfrage setzt derzeit Software-Tools und/oder Checklisten zur Berücksichtigung von Umweltaspekten ein. Von diesem Siebtel wiederum benutzt weniger als ein Zehntel LCA-Software. ${ }^{3}$ Von denen, die das LCA nicht einsetzen wollen, hält ein Fünftel die Nutzung für zu kompliziert. Ein weiteres Siebtel schätzt die Datenqualität als nicht vertrauenswürdig ein. ${ }^{4}$ Damit Umweltaspekte verstärkt in die Gebäudeplanung einfließen können, müssen die Hilfsmittel für deren Berücksichtigung in den üblichen Workflow integrierbar, einfach zu bedienen und kostengünstig zu erwerben sein. Hier besteht noch weiterer Entwicklungsbedarf.

d) Der Wunsch nach Unterstützung durch ökologische Deklarationen oder Zertifizierungen auf hohem Niveau erstreckt sich über alle zum Bauen und Nutzen benötigten Produkte. Ganz besonders hoch ist er bei den Bauwerkstoffen und den Bauhilfsstoffen. Mit dieser Antwort wird ein wesentlicher Bedarf formuliert, den zu befriedigen in erster Linie die Hersteller aufgerufen sind.

e) Wenige wichtige Kennzahlen sollen nach den Wünschen von drei Vierteln der Planer in den Deklarationen ausgewiesen werden. Die Frage an die Umweltschutzpolitik und die Wissenschaft bleibt weiterhin, welche Kennzahlen dies sein könnten bzw. sollten.

f) Die Forderung nach der Angabe von möglichen Ausgasungen in Deklarationen von Bauprodukten, die im Innenraum eingesetzt werden können, ist Konsens bei den Planenden. Insgesamt zeigt die Umfrage einen großen Bedarf an Informationen zu Fragen der Bauteilalterung, der Behaglichkeit, Raumluftqualität und Wohngesundheit, der durch die ausschließliche Anwendung von LCAVerfahren nicht abgedeckt werden kann, jedoch flankierend wichtig ist, um die Akzeptanz und Einsatz solcher Tools zu verbessern. 
Abb.1: Organisation des Informationsflusses

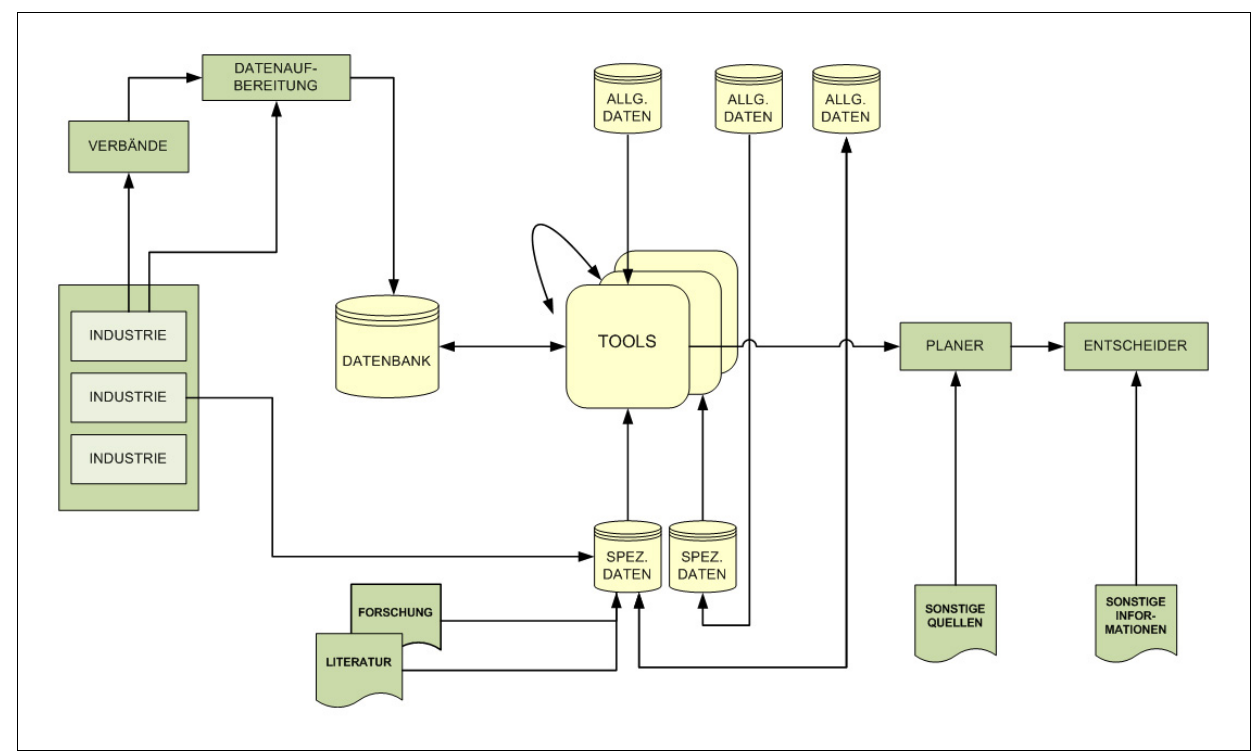

Quelle: Lützkendorf, Zak 2007

\subsection{Gestaltung des Informationsflusses zwischen ausgewählten Akteuren}

Im Rahmen des Netzwerkes Lebenszyklusdaten wurden durch den Arbeitskreis „Nutzersichten im Baubereich“ Anforderungen der Nutzer von LCA-Daten an die Art der Datenbereitstellung nach Art, Qualität und Aggregationsstufe formuliert und Vorschläge für die künftige Gestaltung des Informationsflusses entwickelt. ${ }^{5}$ Die Ergebnisse wurden in einer Studie zusammengefasst (Graubner et al. 2007). Zusätzlich zu den bereits oben diskutierten Akteuren und ihren Rollen in der Informationskette wurde u. a. die Rolle der Verbände und ihre Bedeutung für die Entwicklung von Durchschnittswerten für frühe Phasen der Planung herausgearbeitet (siehe hierzu auch Abb. 1).

\section{Aktuelle Entwicklungen in Deutschland}

Der Bund versucht seit längerem mit zahlreichen Initiativen, den Akteuren der Bauindustrie sowie der Bau- und Immobilienwirtschaft das Thema Nachhaltigkeit und LCA als Methode zur Quantifizierung der Ressourceninanspruchnahme und von Wirkungen auf die Umwelt nahe zu bringen. Das BMVBS hat hierzu u. a. im Jahr 2000 den Leitfaden Nachhaltiges Bauen entwickelt und eingeführt. Mit dem Leitfaden wurde ein Instrument zur Verfügung gestellt, mit dem ganzheitliche Ansätze im Sinne der Nachhaltigkeit bei Bundesbaumaßnahmen sicher umgesetzt werden können. Im Rahmen von Ressortforschungsvorhaben erfolgt derzeit die Fortschreibung und Weiterentwicklung des Leitfadens. ${ }^{6}$

Mit dem Runden Tisch ist ein Gremium zur Unterstützung des BMVBS für Regelungen des Nachhaltigen Bauens des Bundes eingerichtet worden. ${ }^{7}$

Im Rahmen von Aktivitäten des BMVBS und des Bundesamts für Bauwesen und Raumordnung (BBR) werden derzeit erhebliche Anstrengungen unternommen, um eine Integration des LCA in Planungs- und Entscheidungsprozesse zu unterstützen. Dies sind u. a.

a) die Vereinheitlichung von Regeln für die Beschreibung umweltrelevanter Eigenschaften von Bauprodukten und der Bewertung der Umweltqualität von Bauwerken:

Durch Forschungsprogramme und eine Projektförderung wird die intensive Mitwirkung Deutschlands in der internationalen (u. a. ISO TC 59 SC 17) und europäischen (u. a. CEN TC 350) Normung gefördert. Im Ergebnis werden Grundlagen für die Beschreibung und Darstellung umweltrelevanter Informationen zu Bauprodukten in Form von EPDs (environmental product declarations) entstehen, die auf product category rules basieren. Für die Bewertung der Umweltqualität von Bauwerken werden Krite- 
rien, Indikatoren und Regeln definiert, die sich wesentlich auf das LCA abstützen.

b) die Bereitstellung von vereinheitlichten Datengrundlagen

In enger Kooperation mit der Baustoffindustrie wird derzeit an der Entwicklung einer einheitlichen Datengrundlage für die ökologische Bewertung von Bauprodukten gearbeitet. Die dort einzustellenden Daten basieren auf dem Stand der internationalen und europäischen Normung. Die Erstellung der Datenbank wird über eine Projektförderung unterstützt.

c) die Harmonisierung von Planungs- und Bewertungshilfsmitteln auf vereinheitlichter Datenbasis:

Durch die Zusammenführung von Planungsund Bewertungshilfsmitteln durch Herstellen der Kompatibilität und eines Datenaustausches sollen Glaubwürdigkeit und Richtungssicherheit existierender Planungs- und Bewertungshilfsmittel unterstützt werden. In einem vom BBR im Rahmen des Programms „Zukunft Bau“ geförderten Projektes werden Grundlagen für die Zusammenführung von Baustoffinformationssystemen (Bereitstellung von Informationen zur Baustoffbewertung und -auswahl - z. B. ECObis), Pla- nungs- und Bewertungshilfsmitteln für die Konstruktionsoptimierung (z. B. bauloop) sowie für die Unterstützung der Planung und Bewertung von Bauwerken (z. B. LEGEP) in einem System sich arbeitsteilig ergänzender Werkzeuge entwickelt und umgesetzt. ${ }^{8}$ Diese Werkzeuge greifen auf die einheitliche Datengrundlage $\mathrm{zu}$ (s. unter b). In einem weiteren Projekt wird an einer Handlungsanleitung für Planer gearbeitet, um Planern den Zugang zu Informationen, Daten und Hilfsmitteln zu erleichtern und um Fragen der Bewertung der Umweltqualität von Bauwerken $-u$. a. auch auf Basis von Umweltinformationen zu Bauprodukten - in den Planungsablauf zu integrieren (siehe hierzu auch Abb. 2). ${ }^{9}$ Danach konkretisiert sich der Informationsbedarf der Planenden entlang der HOAI-Leistungsphasen von zunächst allgemeinen Daten bzw. Durchschnittsdaten hin zu produktspezifischen Daten bei der Ausschreibung. ${ }^{10}$ Die Informationsquellen für die Tools sind hierbei unter anderem Berichte öffentlicher Institutionen, Berufsgenossenschaften und der Industrie.

d) die Entwicklung von Grundlagen für die Zertifizierung des Beitrages von Einzelbauwerken zu einer nachhaltigen Entwicklung

\section{Abb. 2: Integration von Informationen zur Beurteilung der Umweltqualität in den Planungsprozess}

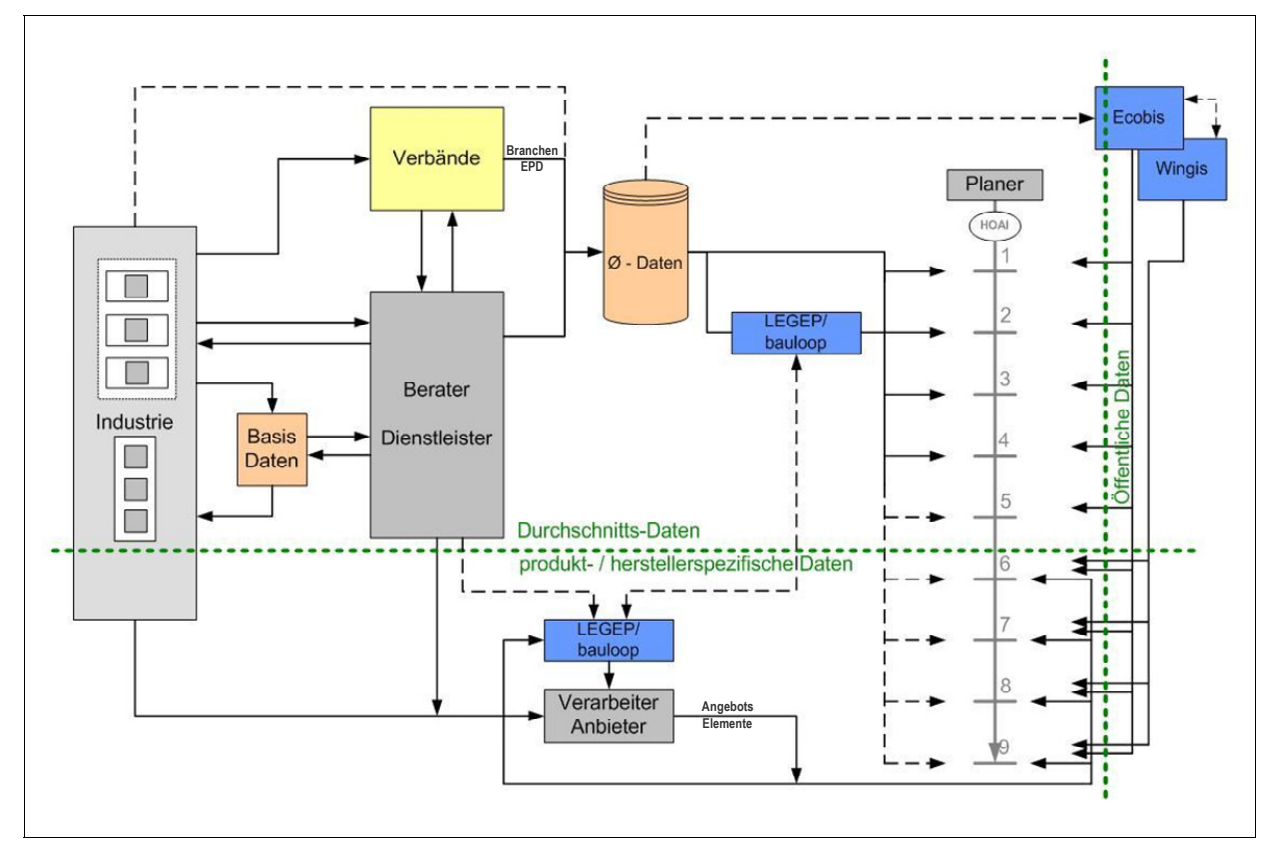

Quelle: Lützkendorf, Zak 2007 
auf der Basis der Festlegungen des Leitfadens Nachhaltiges Bauen:

Durch die TU Darmstadt werden unter Mitwirkung der Universität Karlsruhe derzeit im Rahmen des Forschungsprogramms „Zukunft Bau“ Grundlagen und Vorschläge für die Entwicklung eines Zertifizierungssystems für nachhaltige Gebäude entwickelt. Neben der technischen, funktionalen/sozialen und ökonomischen Performance wird die Umweltqualität beschrieben, bewertet und dargestellt. Die Bewertung der Umweltqualität stützt sich wesentlich auf die Ergebnisse des LCA ab und bezieht die Ergebnisse ein, die unter Verwendung von Planungs- und Bewertungshilfsmitteln (siehe c) erzeugt werden.

e) die Zertifizierung als Grundlage für Investitionsentscheidungen:

In einem von der Universität Stuttgart und der Universität Karlsruhe gemeinsam mit Praxispartnern bearbeiteten Projekt wird derzeit untersucht, ob und inwieweit der Nachweis eines positiven Beitrages von Gebäuden zu einer nachhaltigen Entwicklung die Grundlage für die Entwicklung neuer Produkte für ein Immobilieninvestment sein können. Im Zusammenhang mit der Wahrnehmung einer Verantwortung gegenüber Umwelt und Gesellschaft fragen Anleger vermehrt nachhaltige Geldanlagen nach. Im vom BBR geförderten Projekt wird u. a. untersucht, wie Immobilienfonds ausgestaltet werden könnten, die in ihrem Objektportfolio nachweislich energiesparende, ressourcenschonende und gesundheitsgerechte Gebäude haben.

f) die Weiterentwicklung von Grundlagen für die Aus- und Weiterbildung der am Bau Beteiligten:

Als Beitrag zur UN-Dekade „Education for Sustainable Development" wird im Rahmen des Forschungsprogramms „Zukunft Bau“ durch die Universität Karlsruhe und die TU Darmstadt ein Konzept entwickelt, welches, aufbauend auf einer Analyse von Schlüsselakteuren und Ausbildungskonzepten, Vorschläge für eine stärkere Integration von Grundlagen und Methoden der Beurteilung (u. a. der Umweltqualität in die Aus- und Weiterbildung) entwickeln soll. Neben der Aus- und Weiterbildung von Planern betrifft dies auch und insbesondere Hinweise für die Weiterentwicklung der Lehrangebote u. a. für Führungskräfte der Wohnungswirtschaft, Finanz- und Versicherungswirtschaft, Makler und Wertermittler.

Es wird deutlich, dass sich in der Bauwirtschaft ein System herauszubilden beginnt, welches die Integration des LCA in Entscheidungen relevanter Akteure vorbereitet und unterstützt.

\section{Zu lösende Aufgaben}

Bei dem Prozess, die Ergebnisse aus dem LCA in reale Arbeitsabläufe der Planung und Entscheidungsfindung $\mathrm{zu}$ integrieren, treten noch eine Reihe von Fragen auf. Um die Entwicklung, die sich in dem großen Interesse - insbesondere der öffentlichen Hand - an einer Integration des LCA in die Beschreibung, Bewertung und Darstellung des Beitrages von Einzelbauwerken zu einer nachhaltigen Entwicklung zeigt, weiter zu festigen, bedürfen sie einer raschen Lösung. Dies sind u. a.

a) methodische Fragen bei der Beschreibung und Bewertung der Ressourceninanspruchnahme:

Es besteht i. d. R. das Ziel, neben den Wirkungen auf die Umwelt auch den Energieund Stoffstrom als Teil der Ressourceninanspruchnahme in die Bewertung einzubeziehen. Gewünscht wird im Minimum die Unterscheidung zwischen erneuerbaren und nicht erneuerbaren bzw. zwischen biotischen und abiotischen Ressourcen unter besonderer Beachtung der einer stofflichen bzw. energetischen Nutzung zugeführten Energieträger. Während sich für letztere über den kumulierten Energieaufwand ein Summenparameter etabliert hat, fehlt bisher für übrige stoffliche Ressourcen eine anerkannte Methode für eine zusammenfassende Darstellung.

b) methodische Fragen bei der Beschreibung und Bewertung der Flächeninanspruchnahme:

Im Zusammenhang mit dem Ziel, die weitere Zunahme der Flächeninanspruchnahme zu begrenzen bzw. zu stoppen besteht ein großes Interesse, Art und Umfang der Inanspruchnahme von Flächen zu beschreiben 
und $\mathrm{zu}$ bewerten. Die Nachfrage nach geeigneten Methoden steigt daher an.

c) methodische Fragen der Wichtung und Aggregation von Umweltwirkungen:

Die Nachfrager nach Ergebnissen einer Beschreibung und Bewertung der Umweltqualität von Bauwerken haben bei einem inzwischen gewachsenen Interesse an Ergebnissen eines LCA einen Bedarf an hochaggregierten Informationen. Bisher fehlt jedoch eine in Deutschland allgemein anerkannte Methode für die Wichtung und Aggregation von Wirkungen auf die Umwelt.

d) methodische Fragen der Erarbeitung und Anwendung von Orientierungs-, Ziel- und Grenzwerten:

Für die Bewertung der Umweltqualität von Bauwerken sowohl in der Planung als auch bei einer Zertifizierung werden Maßstäbe in Form von Orientierungs-, Grenz- und Zielwerten benötigt. Es muss dabei sichergestellt werden, dass die Datengrundlage für den Bewertungsvorgang, das Bewertungshilfsmittel sowie für die Bewertungsmaßstäbe identisch ist. Momentan wird im Baubereich der Ansatz einer Bewertung auf der Basis von Referenzgebäuden entwickelt.

e) Fragen der Integration der Umweltqualität in eine Gesamtbewertung:

Es besteht das Ziel, den generellen Beitrag von Einzelbauwerken zu einer nachhaltigen Entwicklung beschreib- und bewertbar zu gestalten. In der derzeitigen Diskussion wird der Ansatz verfolgt, dass bei Erfüllung funktionaler und technischer Anforderungen ökonomische, ökologische und soziokulturelle Aspekte gleichzeitig und gleichberechtigt zu berücksichtigen sind. Dies führt im Moment zu einer Gleichgewichtung der drei Dimensionen ${ }^{11}$ der Nachhaltigkeit.

Die Lösung der beschriebenen Fragestellungen erfordert sowohl intensive Forschungsaktivitäten als auch die Bereitschaft, zu konsensfähigen und praxistauglichen Lösungen beizutragen.

\section{Ausblick}

Im Baubereich hat der Stakeholderdialog zwischen zahlreichen Akteuren und insbesondere auch mit der öffentlichen Hand zu einer hohen Bereitschaft geführt, Ergebnisse des LCA in realen Entscheidungskontexten $\mathrm{zu}$ verwenden und auch Entscheidungen unter Einbeziehung entsprechender Informationen zu treffen. Insofern existiert eine erhebliche Nachfrage nach gesicherten datentechnischen und bewertungsmethodischen Grundlagen. Die Umsetzung von Prinzipien einer nachhaltigen Entwicklung motiviert bzw. zwingt alle Akteure, Wirkungen auf die Umwelt zu beachten, zu quantifizieren und zu berücksichtigen. Die Wissenschaft ist nun aufgerufen, entsprechende Grundlagen und Hilfsmittel noch stärker an Bedürfnisse der Entscheider anzupassen und kann somit ihren Beitrag zur Erfüllung wesentlicher gesellschaftlicher Ziele leisten.

\section{Anmerkungen}

1) Dies geschah u. a in der Arbeitskreis-Sitzung im Netzwerk Lebenszyklusdaten im November 2002.

2) http://www.netzwerk-lebenszyklusdaten.de

3) Allerdings gilt es dabei zu bedenken, dass der Nutzerkreis der Anwender von Ökobilanzierungs-Tools bereits größer ist. Während die hier zugrunde liegende Umfrage nur an die in den Architektenkammern eingetragenen Planer adressiert war, zeigt eine etwas frühere Befragung zu den Wünschen und Bedürfnissen der Nutzer einer frei downloadbaren Bilanzierungssoftware für Gebäude (BEES 2.0 - Building for Environmental and Economic Sustainability), dass weniger als ein Drittel der User dieser Software Planer sind (Hofstetter 2003).

4) Diese reservierte Einstellung wird auch von einer anderen Untersuchung gestützt: Ein Vergleich LCA-basierter Gebäudebewertungsund Planungsinstrumente, welcher neun Ökobilanzierungsprogramme für Gebäude betrachtet, kam ebenfalls zu dem Schluss, dass sowohl eine Harmonisierung und Vereinfachung der Bewertungsinstrumente als auch eine Schulung für die Interpretation der Resultate durch den Planer notwendig sei. Außerdem müssten viele Programme transparenter werden (Kellenberger 2004).

5) Der Arbeitskreis wird getragen von der TU Darmstadt, der Universität Karlsruhe sowie der Fachhochschule Augsburg und wird unterstützt vom Forschungszentrum Karlsruhe.

6) Für 2008 ist die Veröffentlichung eines weiterentwickelten Leitfadens geplant, welcher dann neben Hinweisen für die Planung von Neubauten auch Vorgaben für das Planen und Bauen im Bestand sowie für die Beschreibung und Bewertung 
des Beitrages von Gebäuden zu einer nachhaltigen Entwicklung enthalten wird.

7) Zum Runden Tisch siehe http://www.bbr.bund. de/nn 22252/DE/ForschenBeraten/Bauwesen/ NachhaltigesBarrierefreiesBauen/Nachhaltiges Bauen/runderTisch/RunderTisch.html.

8) Das Projekt mit dem Titel „Zusammenführung und Harmonisierung vorhandener Planungs- und Bewertungsmittel zu einem Gesamtsystem“ wird von der TU Darmstadt und der Universität Karlsruhe $(\mathrm{TH})$ koordiniert und ist eines der Themen zum Forschungscluster Bauqualität in der Auftragsforschung des laufenden Forschungsprogramms „Zukunft Bau“ des Bundesamtes für Bauwesen und Raumordnung http://www.bbr. bund.de/nn_112742/DE/Forschungsprogramme/ ZukunftBau/Auftragsforschung/auftragsfor projekte_20in_20bearbeitung.html.

9) Bei diesem Teilprojekt aus dem Forschungsprogramm ,Zukunft Bau“ mit dem Titel ,Entwicklung von Handlungsanleitungen für Planer“ kooperieren das Architekturbüro ,sol-id-ar Berlin“ und die Universität Karlsruhe (TH).

10) HOAI ist die Honorarordnung für Architekten und Ingenieure zur Regelung der Vergütung der Leistungen von Architekten und Ingenieuren in Deutschland.

11) Die Enquete-Kommission des 13. Deutschen Bundestages - Schutz des Menschen und der Umwelt - Ziele und Rahmenbedingungen einer nachhaltig zukunftsverträglichen Entwicklung sieht für das Leitbild einer nachhaltig zukunftsverträglichen Entwicklung drei Dimensionen, für die sie jeweils grundlegende Regeln vorschlägt, ableitet oder von anderen übernimmt: 1 . die ökologische Dimension, 2. die ökonomische Dimension und 3. die soziale Dimension.

\section{Literatur}

BBR - Bundesamt für Bauwesen und Raumordnung, 2001: Leitfaden Nachhaltiges Bauen. Berlin: Bundesministerium für Verkehr, Bau- und Wohnungswesen

CEN TC 350: Sustainability of construction works. Laufendes Normungsprojekt des Europäischen Komitees für Normung, Informationen unter: http:// www.cen.eu/CENORM/BusinessDomains/Technical CommitteesWorkshops/CENTechnicalCommittees/ CENTechnicalCommittees.asp?param $=481830 \&$ title $=\mathrm{CEN} \% 2 \mathrm{FTC}+350$; download 14.12.2007

Graubner, C.-A.; Schneider, C.; Renner, A. et al., 2007: Formulierung von Anforderungen an die Bereitstellung von Ökobilanzdaten nach Art, Qualität und Aggregationsstufe, Studie des AK Nutzersichten im Baubereich des Netzwerkes Lebenszyklusdaten. Darmstadt, Karlsruhe, Augsburg
Hofstetter, P.; Mettier, Th.M., 2003: What Users Want and May Need: Insights from a Survey of Users of a Life-Cycle Tool. In: Journal of Industrial Ecology 7/2 (2003), pp. 79-101

ISO TC 59 SC17: Sustainability in building construction. Laufendes Normungsprojekt der internationalen Normungsorganisation, Informationen unter http:// www.iso.org/iso/fr/iso_technical_committee?commi $\mathrm{d}=322621$

Kellenberger, D., 2004: Vergleich LCA-basierter Gebäudebewertungs- und Planungsinstrumente in: 13. Schweizerisches Status-Seminar 2004; v/b, Verlag Zentrum für Energie und Nachhaltigkeit im Bauwesen, EMPA Dübendorf, S. 445-454

Klingele, M.; Jeske, U., 2007: Umweltaspekte und Lebenszyklusdaten in der Gebäudeplanung. OnlineUmfrage unter ArchitektInnen und PlanerInnen. Band 1: Kumulierte Ergebnisse. ITC-ZTS; http://www.netzwerk-lebenszyklusdaten.de/umfrage

Lützkendorf, T.; Zak, J., 2007: Informationsflüsse im Baubereich - Begrifflichkeiten, Analyse der gegenwärtigen Situation und Systematik beteiligter Akteure, Teilbericht zum Forschungsvorhaben Nutzersichten im Baubereich - Formulierung von Anforderungen an die Datenbereitstellung nach Art, Qualität und Aggregationsstufe, Karlsruhe 2007

Rogall, H., 2004: Ökonomie der Nachhaltigkeit Handlungsfelder für Politik und Wirtschaft. Wiesbaden

\section{Kontakt}

Dr. Martina Klingele

ITC-ZTS im Forschungszentrum Karlsruhe

Hermann-von-Helmholtz-Platz 1,

76344 Eggenstein-Leopoldshafen

Tel.: +49 (0) 7247 / 82 - 8331

martina.klingele@itc-zts.fzk.de

Prof. Dr.-Ing. habil. Thomas Lützkendorf

Universität Karlsruhe (TH)

Lehrstuhl Ökonomie und Ökologie

des Wohnungsbaus

Postfach 69 80, 76128 Karlsruhe

thomas.luetzkendorf@wiwi.uni-karlsruhe.de 